\title{
Benefit of hearing aid use in the elderly: the impact of age, cognition and hearing impairment
}

\author{
Il beneficio protesico e il ruolo delle abilità cognitive, dell'età e del deficit uditivo \\ nel paziente anziano \\ G. TOGNOLA ${ }^{1}$, A. MAINARDI ${ }^{2}$, V. VINCENTI ${ }^{3}$, D. CUDA² \\ ${ }^{1}$ Consiglio Nazionale delle Ricerche, Istituto di Elettronica e di Ingegneria dell'Informazione e delle \\ Telecomunicazioni (CNR IEIIT), Milan, Italy; ${ }^{2}$ Ospedale "Guglielmo da Saliceto", UO Otorinolaringoiatria, Piacenza, \\ Italy; ${ }^{3}$ Università degli Studi di Parma, Clinica Otorinolaringoiatrica, Parma, Italy
}

\section{SUMMARY}

This study describes the benefits of hearing aid (HA) use in a sample of elderly experienced HA users ( $\mathrm{n}=102$, mean age 81.1 years), investigating the role of the age, hearing impairment and cognitive function. The benefit was assessed in aided condition by measuring audiometric outcomes (aided thresholds and speech reception in quiet and in noise) and self-assessed outcomes addressing the disability and the handicap domains of auditory dysfunction. Several cognitive abilities were assessed, including short-term memory, working memory and executive functions. To discover potential latent factors and assess which factors significantly influenced the benefit of HA use, age, hearing impairment, cognitive function, audiometric and self-assessed outcomes were examined with multivariate analysis, followed by correlation and regression analysis. The analysis revealed a significant improvement in aided audiometric outcomes and a decrease in the perceived disability and handicap in the sample population. Multivariate, correlation and regression analyses showed that better aided audiometric outcomes were significantly associated with hearing impairment of lower degree and higher cognitive abilities. Moreover, self-assessed outcomes were significantly associated with audiometric outcomes and hearing impairment: subjects with better audiometric outcomes and lower hearing impairment tend to perceive more benefit in using their HAs. Cognition was only indirectly associated with self-assessed outcomes through its direct correlation with aided audiometric outcomes, meaning that the perceived subjective benefit with HA was not directly associated with better cognitive abilities.

KEY WORDS: Elderly $\bullet$ Hearing impairment $\bullet$ Speech reception threshold $\bullet$ Montreal cognitive assessment $\bullet$ Auditory disability

\section{RIASSUNTO}

Questo studio analizza il beneficio protesico in un campione di soggetti anziani ( $n=102$, età media 81,1 anni), indagando il ruolo svolto dall'età, dal deficit uditivo e dalle abilità cognitive. Il beneficio protesico è stato valutato sia come miglioramento delle caratteristiche audiometriche con protesi (in termini di soglie tonali e soglie di percezione del parlato in quiete e nel rumore) sia come diminuzione della percezione della disabilità e dell'handicap dovuti alla ipoacusia. Sono state valutate diverse abilità cognitive, tra cui la memoria a breve termine, le funzioni esecutive e l'attenzione. I dati raccolti sono stati analizzati tramite un'analisi multivariata, seguita da un'analisi di correlazione e regressione per evidenziare possibili fattori latenti tra le variabili in gioco e valutare come l'età, il deficit uditivo e le abilità cognitive influenzavano il beneficio protesico. L'analisi ha rivelato un miglioramento significativo del profilo audiometrico con protesi e una diminuzione della disabilità e dell'handicap percepiti. L'analisi multivariata, insieme con quella di correlazione e di regressione ha evidenziato che i soggetti con migliori abilità cognitive e minor deficit uditivo erano significativamente caratterizzati da un più evidente miglioramento del profilo audiometrico con protesi. Inoltre, è stata rilevata una relazione significativa tra la disabilità e l'handicap percepiti, il profilo audiometrico con protesi e il grado di ipoacusia: i soggetti con miglior profilo audiometrico con protesi e minor deficit uditivo erano significativamente caratterizzati da una più evidente diminuzione dell'handicap e della disabilità percepiti. Lo studio non ha invece evidenziato alcuna relazione diretta tra le abilità cognitive e il beneficio protesico riguardante la percezione dell'handicap e della disabilità.

PAROLE CHIAVE: Anziano $\bullet$ Ipoacusia $\bullet$ Disabilità uditiva $\bullet$ Abilità cognitive $\bullet$ Percezione del parlato

\section{Introduction}

According to the 2012 World Health Organization estimates ${ }^{1}$, about one-third of persons above 65 years are affected by disabling hearing loss. Current reviews (see, e.g. Fortunato et al. ${ }^{2}$ ) highlight that hearing loss can af- fect cognitive performance by reducing and worsening communication skills, thus leading to diminished social interactions and depression. On the other hand, the same studies revealed that impaired cognitive performance may exacerbate the detrimental effects of hearing loss on the quality of life. It is clear that hearing loss and 
cognitive abilities are somewhat linked and influence each other. In this context, as hearing aids (HA) help in providing better auditory inputs in the hearing impaired, it is important to gain more insights both on the possible role of HAs in lowering cognitive decline and, vice versa, on the effects that cognitive abilities have on the benefits that a hearing impaired subject could gain by using the HA. This study addressed the latter of the two topics, that is, how cognitive skills could influence the benefit achievable with an HA. In fact, evidence suggests that hearing benefit with HA use is influenced by many factors, including cognitive skills ${ }^{3-8}$. As cognitive abilities naturally tend to decrease with age ${ }^{9}$, the assessment of the real HA benefit in the elderly is a crucial research topic ${ }^{10-12}$. Although there is evidence that HA use in the elderly is beneficial in diminishing hearing handicap and improving the quality of life, hearing performance in aided conditions was found to differ widely across elderly HA users.

In most studies, HA benefit was investigated using either clinical audiometric outcomes or self-assessed outcomes. Only a few studies 478 have investigated HA benefit using a 'multi-parametric' experimental protocol that considered the main factors that may have a role in HA benefit, i.e., including both audiometric and selfassessed outcomes, age, cognitive skills and hearing impairment. These latter studies have provided valuable insights on the factors correlated with HA benefit in the elderly, However, the data remains limited and due to the vast nature of the research topic the evidence is not conclusive and needs to be further explored. Moreover, these studies implemented experimental protocols that are difficult to be replicated in typical clinical settings and clinical routines as they used batteries of tests suitable for a research laboratory (requiring, e.g., demanding tests of long duration). Last but not least, all of them but Meister et al. ${ }^{7}$ also included subjects younger than 65 years and thus their results cannot be strictly associated with the aged population, which is the focus of our work.

The aim of the present study is to further extend the current knowledge of HA benefit in the elderly by using: (1) an easy to be used and replicated multi-parametric clinical protocol that simultaneously assessed all the main factors potentially contributing to the benefit, i.e., audiometric and self-assessed outcomes, age, cognitive skills and hearing impairment; (2) an ample (> 100 subjects) sample of elderly HA users (greater than 65 years); (3) advanced statistical analysis based on multivariate analysis, correlation analysis and regression analysis to discover possible factors between the measured variables and investigate which variables most in- fluence the benefit obtained in using the HA, and how they were correlated each other; (4) last but not least, different from previous studies, the current study evaluated self-assessed outcomes addressing both of the two domains of auditory dysfunction, i.e., the disability (e.g., the quality of communication) and handicap domains (e.g., emotional problems due to hearing impairment).

\section{Materials and methods}

\section{Recruiting criteria}

Experienced HA users after at least one year from the first fitting, aged $\geq 65$ years, with mono- or bilateral-amplification were recruited. Eligible participants were identified from the HA registry of the Local Unit of the National Health System which provided total or partial coverage of the HA costs. The presence of other diseases or health conditions were not considered factors for exclusion. The local Institutional Review Board approved all procedures; all participating subjects provided written consent.

\section{Experimental protocol}

The protocol included a battery of audiometric tests and questionnaires that can be easily used and adopted in clinical routine and aimed at evaluating audiometric outcomes, self-assessed outcomes and cognitive skills. The tests were conducted at the outpatient service of the ENT Department of the Hospital "Saliceto". Audiometric outcomes were assessed in sound-field and aided conditions through the measurement of thresholds to warble tones in the $250-4,000 \mathrm{~Hz}$ range and speech reception threshold (SRT) in quiet and in noise (SRTnoise) in a soundproof room. For SRT in quiet, speech stimuli (60 disyllabic words) ${ }^{13}$ were pre-recorded and delivered by a loudspeaker at $65 \mathrm{~dB}$ SPL at zero degrees azimuth in the sound field. The speech reception threshold, defined as the level of speech corresponding to $50 \%$ of correct answers, was then determined. SRT-noise was performed through the Italian matrix sentence test ${ }^{14} 15$. The test was administered using the SONO presentation setup (speech and noise from the same frontal loudspeaker). SRT in noise was defined as the signal-tonoise $(\mathrm{S} / \mathrm{N})$ ratio at which the subject scored correctly $50 \%$ of words. The background noise was presented at $65 \mathrm{~dB}$ SPL, whereas the speech level was adaptively adjusted depending on the subjects' response to obtain the $\mathrm{S} / \mathrm{N}$ ratio at which the percent word score was $50 \%$. Two lists of 30 sentences, having a fixed syntactic structure, were generated by picking up items from a 105-word base matrix ${ }^{14}$. Before testing, a training list 
was applied. The test was conducted using a closed-set response format.

Self-assessed outcomes were measured with a battery of three inventories: IOI-HA ${ }^{16}$, HHIE-S ${ }^{17}$ and APHAB ${ }^{18}$. IOI-HA and APHAB address mostly the disability (e.g., the quality of communication) domain of auditory dysfunction, whereas HHIE-S mainly addresses the handicap domain (e.g., emotional problems due to hearing impairment).

IOI-HA evaluates the perceived effectiveness of HA treatment and targets seven different outcome domains, ranging from the perceived benefit with HA to the residual activity limitations. A high score is an indication of a good outcome in aided conditions. HHIE-S assesses the perceived situational difficulties due to hearing impairment in the elderly people and explores the social/situational impact and the emotional consequences of hearing impairment. A low HHIE-S score is indication of less perceived difficulties. APHAB assesses the difficulty in speech understanding in several typical workday situations. The lower the score, the better the performance. Participating subjects were asked to score IOI-HA and HHIE-S questionnaires regarding the difficulties they perceived in the aided condition. Instead, for APHAB, participants were asked to score the questionnaire items both in the unaided and aided conditions; a measure of the benefit was calculated by subtracting APHAB scores in the aided from those in the unaided condition.

Cognitive skills were assessed with the MOCA test ${ }^{19}$, which is a screening tool for mild cognitive impairment consisting of 12 subtasks assessing different cognitive skills, including short-term and delayed verbal memory, executive functions, and attention. The total MOCA score ranges from 0 (worst performance) to 30 (best performance).

Finally, hearing impairment was assessed for the right and left ears with pure tone audiograms at the audiometric frequencies $125-8,000 \mathrm{~Hz}$ in a sound-proof room with a Madsen Astera audiometer and TDH39 supraaural earphones.

\section{Data analysis}

Following Cox et al. ${ }^{20}$, IOI-HA scores were obtained separately for subjects with mild-moderate and severeprofound hearing impairment. Scores for the two groups were then compared to the normative data from bilaterally fitted adults ${ }^{20}$. Differences in group means between our subjects and the normative group were tested by t-test with significance level $\mathrm{p}<0.01$; differences were tested both assuming unequal variances (by using the WelchSatterthwaite procedure) and equal variances (using the pooled standard deviation procedure) between the groups.
As to HHIE-S analysis, the distribution of the scores in the aided condition was obtained as a function of the hearing impairment for the three HHIE handicap categories defined in Lichtenstein et al. ${ }^{21}$, i.e., "no handicap" (HHIE-S: 0-8), "mild handicap" (HHIE-S: 10-22), and "significant handicap" (HHIE-S: 24-40).

As to APHAB analysis, scores in unaided condition were compared to those in the aided one for each questionnaire subscale; Wilcoxon paired t-test was used to compare differences in group means between the unaided and aided conditions (significance level $\mathrm{p}<0.01$, two tails).

Following Santangelo et al. ${ }^{22}$, the raw total scores of the MOCA test were adjusted to be compared to the Italian norm obtained from a sample population with no current or past history of neurologic or psychiatric diseases and aged 21-95 years. The score adjustment was computed as follows ${ }^{22}$ :

$$
\begin{aligned}
& \text { adjusted }_{\text {MoCA }_{\text {score }}} \\
& \quad=\text { raw }_{\text {MocA }} \text { score } \\
& \\
& *[\sqrt{\text { years_education }}-3.25]
\end{aligned}
$$

where raw $_{\text {MoCAscore }}$ is the total MOCA score calculated as the sum of the scores obtained at the 12 tasks of the test, age is the subject age in years and years_education are the number of years spent at school/university. The adjusted MOCA scores were then mapped to a five-point interval scale ranging from 0 to 4 Equivalent Scores (ES), as defined in Santangelo et al. ${ }^{22}$. In this new scale, ES $=0$ is for adjusted scores $\leq 5 \%$ the outer tolerance limit (which is set as the threshold for an abnormal performance and is suggestive for a cognitive decline), whereas ES $=4$ is for scores higher than the median values of the normative sample. ES from 1 to 3 are for intermediate scores between the threshold for pathology $(\mathrm{ES}=0)$ and the median value $(\mathrm{ES}=4)$.

\section{Statistical analysis}

Statistical analysis was divided in two sequential steps. Step \#1 was aimed at investigating how hearing impairment, cognitive skills, patient age and HA use influenced the audiometric outcomes in aided condition. Step \#2 was aimed at understanding how self-assessed outcomes were related and might be predicted by the audiometric outcomes measured in aided condition, hearing impairment, cognitive skills, patient age and HA use.

Both investigations applied multivariate exploratory factor analysis to reduce the number of variables and identify possible latent factors, followed by Spearman correlation to analyse the relations between the variables and 
the factors, and finally regression analysis to investigate how factors and variables relate to each other and how they predict outcomes. Statistical analysis was implemented with the IBM SPSS Statistics (ver. 24) software. Factor analysis was implemented with the principal component method to extract factors with an eigenvalue larger than 1.0; factor scores were calculated with the regression method. Factor analysis was considered appropriated when the Kaiser-Meyer-Olkin measure (KMO) was greater than 0.60 and the Bartlett's was significant at $\mathrm{p}<0.001$. Correlation analysis was performed at a significant level $\mathrm{p}<0.10$ (two tails). Finally, stepwise linear regression analysis with forward selection and entry criterion $\mathrm{p}<0.10$ was performed.

\section{Results}

The recruited participants were 102 elderly hearingimpaired HA users (mean age 81.1 years (SD 6.9); age range 68-97 years; 57 females, 45 males) diagnosed with sensorineural hearing loss. Figure 1 displays mean audiograms for the right and left ears: subjects had a typical age-related hearing loss configuration.

The degree of hearing impairment was then calculated as the four frequency average hearing loss (4FAHL) of the better ear at 500, 1,000, 2,000 and 4,000 Hz. More than half of subjects (52\%) had severe hearing impairment (4FAHL: 61-80 dB HL), $42 \%$ moderate hearing impairment (41-60 dB HL) and 6\% profound impairment (> $80 \mathrm{~dB}$ HL). The average duration of HA use for the entire group was $11.0 \mathrm{~h} /$ day (SD 4.2); $95 \%$ of subjects used the HA for at least $4 \mathrm{~h} /$ day and $23 \%$ for at least $8 \mathrm{~h} /$ day. As for cognitive skills, 94 of 102 subjects had an ES greater than 1 , corresponding to cognitive abilities above the threshold for pathology; 48 of these 94 subjects were in the ES class 4, meaning that their MOCA scores were higher than the median values of the normative sample. The remaining 8 subjects out of 102 are classified in the ES class 0 , indicating that their scores were lower than the threshold for pathology.

\section{Aided audiometric outcomes}

Figure 2 shows the mean aided thresholds in sound-field conditions: the aided threshold was within the long-term average speech spectrum level at $65 \mathrm{~dB}$ SPL.

The distribution of the aided SRT in quiet is reported in Figure 3: for nearly half of subjects (40\%) SRT was within $50 \mathrm{~dB} ; 31 \%$ of subjects had an SRT in the 51$60 \mathrm{~dB}$ range, $14 \%$ in the $61-70 \mathrm{~dB}$ range and $15 \%$ greater than $70 \mathrm{~dB}$.

As to the reception of speech in noise in aided condi-

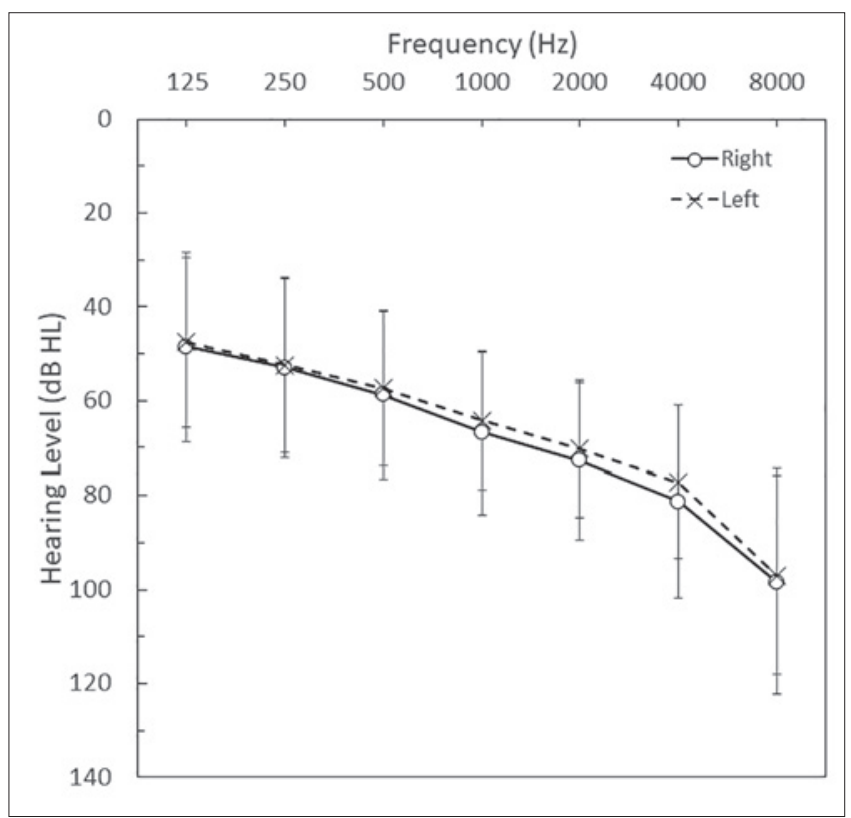

Fig. 1. Mean $( \pm S D)$ air-conduction pure-tone thresholds for the right and left ears.

tion, Figure 4 shows the distribution of $\mathrm{S} / \mathrm{N}$ ratio corresponding to a word score of $50 \%$. Negative values of $\mathrm{S} / \mathrm{N}$ denote better performance: in fact, this means that the patient reached $50 \%$ of correct word recognition when noise was louder than speech. For all subjects, the $\mathrm{S} / \mathrm{N}$ ratio was positive. In particular, in more than half of subjects (62 of 102) the S/N ratio was within $+10 \mathrm{~dB}$, whereas in the remaining 40 subjects $(39.21 \%)$ the $\mathrm{S} / \mathrm{N}$ ratio was greater than $+10 \mathrm{~dB}$.

\section{Self-assessed outcomes}

Table I displays group scores for the seven IOI-HA items for subjects with mild-moderate and severe-profound hearing impairment and the score differences with the IOI-HA normative data by Cox et al. ${ }^{20}$. Our group with mild-moderate hearing impairment had mean scores significantly better than the norm $(\mathrm{p}<0.01$, assuming both unequal and equal variances in our group and the norm) for all IOI-HA items; the severe-profound hearing impairment group had mean scores better than the norm ( $\mathrm{p}<0.01$, assuming both unequal and equal variances in our group and the norm) for items \#5 ("residual participation restriction") and \#6 ("impact on others") and equal to the norm for all the remaining items.

The average HHIE-S score for the entire sample was 13.69 (SD 11.41), indicating a perceived residual handicap of mild degree (HHIE-S: 10-22). As seen in Table II, most subjects (76 of 102) reported a mild or non-sig- 


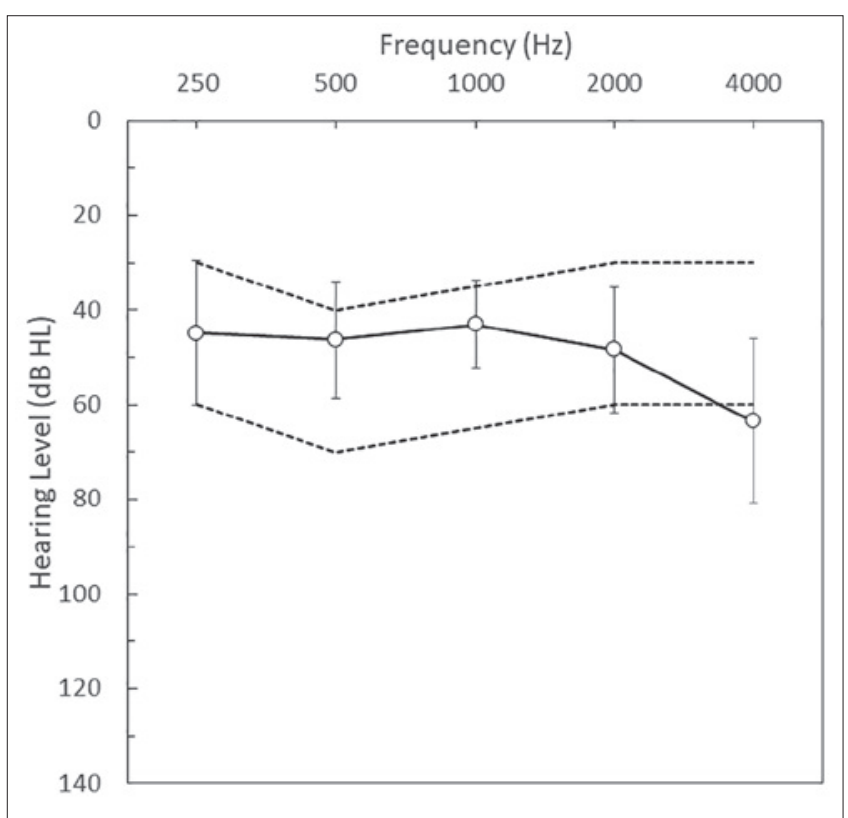

Fig. 2. Mean $( \pm S D)$ aided thresholds in sound field. The dotted lines show the $30 \mathrm{~dB}$ dynamic range of a speech at long-term level of $65 \mathrm{~dB}$ SPL.

nificant handicap in the aided condition, whereas a few subjects (26 of 102) still reported a significant handicap. In particular, 38 of 43 subjects with moderate hearing impairment reported mild or non-significant residual handicap in the aided condition. Those with a severe impairment scored almost equally in all the three HHIE-S

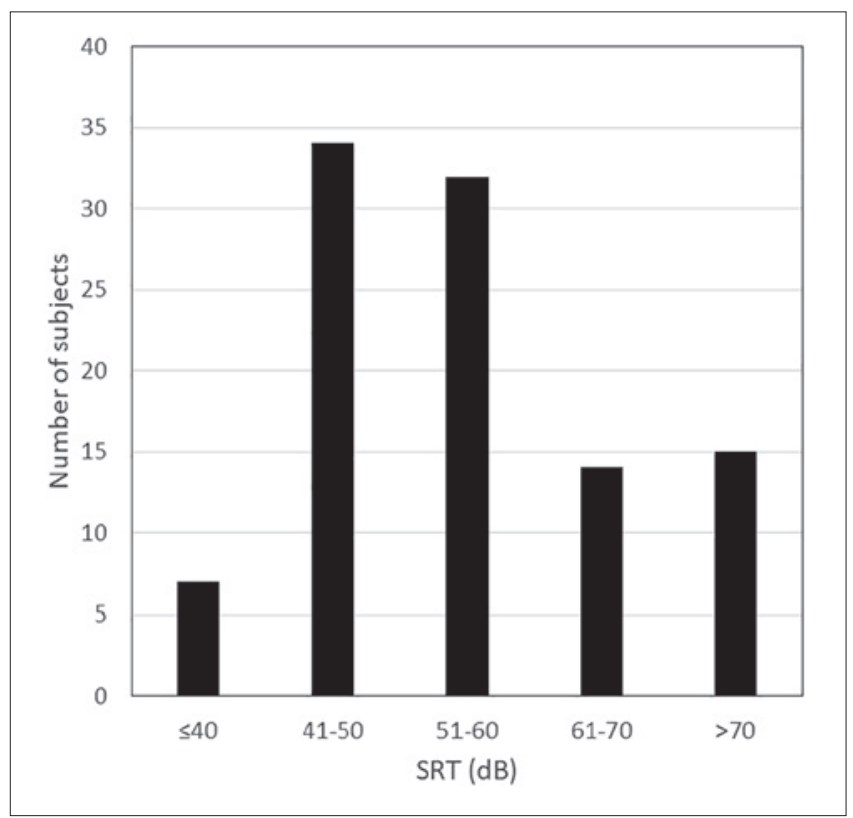

Fig. 3. Aided SRT in quiet. handicap categories and, finally, half of the subjects with a profound impairment reported a mild or non-significant handicap in the aided condition.

On average, the APHAB global score decreased from 82.16 (SD 12.53) in the unaided condition to 37.52 (SD 21.38 ) in the aided condition, indicating very good improvement following HA amplification. Figure 5 shows the scores of the APHAB subscales in aided and unaided conditions. For EC, RV and BN subscales, scores obtained in the aided condition were lower than in the unaided condition, meaning that the subjects perceived less problems in the aided condition compared to the unaided one; for AV subscale, the scores in the aided condition were slightly higher than in the unaided condition. For all subscales, Wilcoxon paired t-test on mean indicated that the difference in the mean scores between the unaided and aided conditions was significant (two-tails, $\mathrm{p}<0.001$ ).

\section{Step \#1 of statistical analysis:}

study of variables affecting aided audiometric outcomes As described in the Methods, step \#1 aimed at assessing, through multivariate factor analysis and correlation and regression analysis, how the aided audiometric outcomes were influenced by hearing impairment (4FAHL), cognitive skills (MOCA scores), patient age and HA use. We used the following audiometric outcomes variables: the average aided threshold at 500, 1,000, 2,000 and 4,000 Hz in sound-field, SRT and SRT-noise. First,

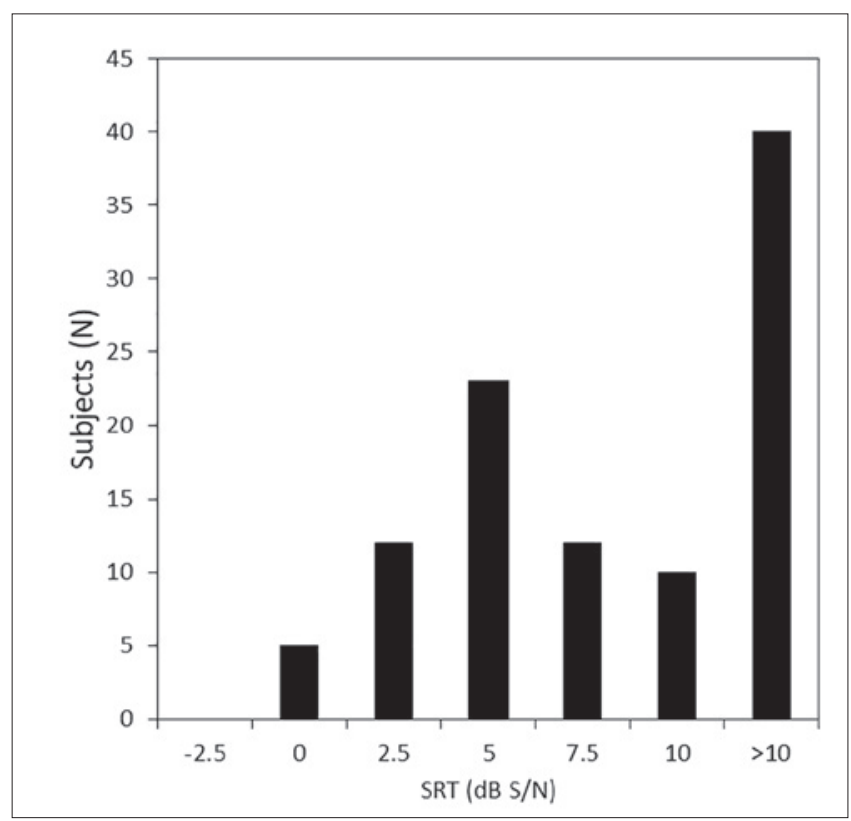

Fig. 4. Aided SRT (dB S/N) for speech reception in noise. 
Table I. Mean (and SD) of IOI-HA scores for subjects with mild-to-moderate and severe-to-profound hearing impairment, as determined by the unaided 4FAHL at the better ear.

\begin{tabular}{|c|c|c|c|c|}
\hline \multirow[t]{2}{*}{ IOI-HA item } & \multicolumn{2}{|c|}{$\begin{array}{l}\text { Mild-moderate } \\
\quad \mathrm{N}=43\end{array}$} & \multicolumn{2}{|c|}{$\begin{array}{l}\text { Severe-profound } \\
\quad N=59\end{array}$} \\
\hline & Mean (SD) & Delta $^{* *}$ & Mean (SD) & Delta(†) \\
\hline 1. Use & $4.60(0.79)$ & $0.87^{*}$ & $4.68(0.75)$ & 0.018 \\
\hline 2. Benefit & $4.09(1.00)$ & $0.70^{*}$ & $3.69(1.29)$ & 0.17 \\
\hline 3. Residual activity limitation & $4.07(0.86)$ & $0.67^{*}$ & $3.63(1.10)$ & 0.44 \\
\hline 4. Satisfaction & $4.09(0.92)$ & $0.89^{*}$ & $3.58(1.19)$ & -0.26 \\
\hline 5. Residual participation restriction & $4.53(0.80)$ & $0.96^{*}$ & $4.07(1.19)$ & $0.69^{\star}$ \\
\hline 6. Impact on others & $4.53(0.93)$ & $0.74^{*}$ & $4.20(1.26)$ & $0.82^{*}$ \\
\hline 7. Quality of life & $4.35(0.78)$ & $1.16^{*}$ & $3.90(0.98)$ & 0.22 \\
\hline
\end{tabular}

$":$ delta is the difference between the group mean scores of our study participants and norms data; a positive delta means that scores of study participants were higher than the norm; ": significant difference $(p<0.01)$.

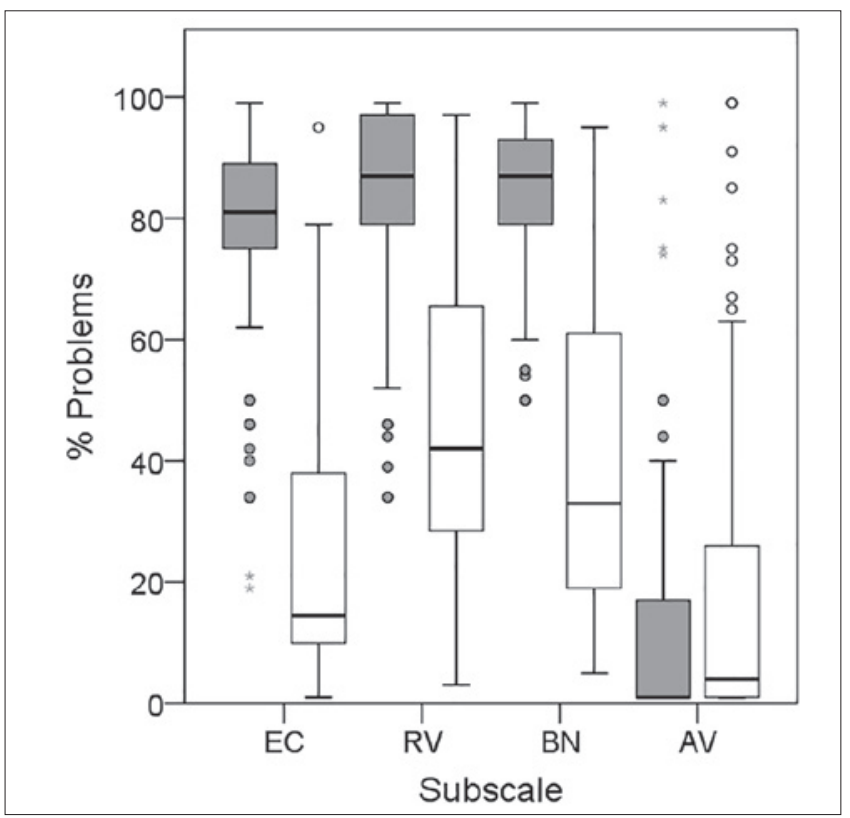

Fig. 5. Boxplot of APHAB scores for unaided (gray rectangles) and aided (white rectangles) conditions for the four subscales EC, RV, BN, and AV $(\mathrm{EC}=$ Ease of Communication; $\mathrm{RV}=$ Reverberation; $\mathrm{BN}=$ Background Noise; $\mathrm{AV}=$ Aversiveness). Thick lines in the boxes are the median value; the bottom and top boundaries of the boxes are the $25^{\text {th }}$ and $75^{\text {th }}$ percentiles; T-bars from boxes extend 1.5 times the height of the box; circles are outliers that fall outside the T-bars; asterisks are extreme outliers (greater than three times the height of the boxes).

multivariate factor analysis was applied to discover potential latent factors among the audiometric outcome variables. It resulted in a one factor solution, with an eigenvalue $>1$, which explained $74 \%$ of variance. The three audiometric outcome variables load almost equally on the single factor solution (Table III).

The correlation analysis revealed that the audiometric outcomes were significantly correlated to hear-
Table II. Distribution of subjects by hearing impairment and HHIE-S handicap categories assessed in aided condition.

\begin{tabular}{lccc} 
& \multicolumn{3}{c}{ HHIE-S handicap categories } \\
Hearing impairment & No handicap & Mild & Significant \\
No impairment & 0 & 0 & 0 \\
Slight & 0 & 0 & 0 \\
Moderate & 23 & 15 & 5 \\
Severe & 21 & 13 & 19 \\
Profound & 1 & 3 & 2 \\
Total subjects & 45 & 31 & 26 \\
\hline
\end{tabular}

ing impairment $(\mathrm{r}=0.400, \mathrm{p}<0.01$, two tails $)$, patient age $(\mathrm{r}=0.367, \mathrm{p}<0.01$, two tails $)$ and cognitive skills $(\mathrm{r}=-0.182, \mathrm{p}<0.1$, two tails $)$, meaning that better audiometric outcomes were associated with lower age, lower hearing impairment and higher MOCA scores. HA use was not associated with any of the variables considered. Regression analysis was then performed between audiometric outcomes and hearing impairment, cognitive skills, patient age and HA use considered as the independent variables. A forward selection with entry criterion $\mathrm{p}<0.10$ was implemented. The analysis confirmed that HA use did not contribute to the overall regression model $(p=0.23)$, whereas the remaining independent variables significantly predicted audiometric outcomes $\left(\mathrm{R}^{2}=0.418\right.$, adjusted $\mathrm{R}^{2}=0.400, \mathrm{~F}(3.98)=23.461$, $\mathrm{p}<0.001)$. As summarised in Table IV, all predictor variables were highly significant $(\mathrm{p}<0.05)$. Table IV also displays for each predictor, the value of the corresponding coefficient (standardised version) in the regression model, which is a measure of how much each predictor variable contributes to the regression model. It is seen that hearing impairment gave the greatest contribution to the audiometric outcomes $(0.497)$, followed by the sub- 
Table III. Factor loadings of multivariate factor analysis on audiometric outcomes and self-assessed outcomes.

\begin{tabular}{ll} 
Test variables & Factor loadings \\
Audiometric outcomes & \\
SRT & 0.915 \\
4 frequency average aided threshold in sound-field & 0.851 \\
SRT-noise & 0.805 \\
Self-assessed HA outcomes & \\
APHAB & 0.916 \\
HHIE & 0.900 \\
IOI-HA & -0.866 \\
\hline
\end{tabular}

$:$ KMO was 0.670 (i.e., above the typical recommended value of 0.6) and the Bartlett's test was significant $\left(\chi^{2}(3)=119.03, p<0.001\right)$; ": KMO was 0.727 and the Bartlett's test was significant $\left(\chi^{2}(3)=153.761, p<0.001\right.$.

ject's age (0.334). Cognitive skills contributed to a less degree $(-0.193)$, nearly half of the contribution of hearing impairment. The regression model of Table IV shows that better cognitive skills (higher MOCA scores), lower hearing impairment and lower age contributed to better audiometric outcomes.

\section{Step \#2 of statistical analysis:}

study of variables affecting self-assessed outcomes

Investigation \#2 aimed at understanding how self-assessed outcomes were related and might be predicted by the audiometric outcomes in aided condition, hearing impairment, cognitive skills, patient age and HA use. As selfassessed outcomes variables we used APHAB, HHIE, and IOI-HA scores. First, a multivariate factor analysis was performed to discover potential latent factors among the self-assessed outcome variables. It resulted in a one factor solution with an eigenvalue $>1$ that explained $80 \%$ of variance; as seen in Table III, APHAB, HHIE and IOI-HA load almost equally on the factor solution.

The correlation analysis revealed that self-assessed outcomes were significantly correlated to audiometric outcomes ( $\mathrm{r}=0.283, \mathrm{p}<0.01$, two tails), hearing impairment $(\mathrm{r}=0.326, \mathrm{p}<0.01$, two tails $)$ and patient age $(\mathrm{r}=0.175, \mathrm{p}<0.1$, two tails $)$.

Table IV. The regression model between audiometric outcomes and predictors, i.e., hearing impairment (4FAHL), cognitive skills (MOCA scores) and patient age.

\begin{tabular}{lcc} 
Predictors & Standardised coefficients & $\mathbf{p}$ \\
Constant & - & 0.000 \\
Hearing impairment & 0.497 & 0.000 \\
Age & 0.334 & 0.000 \\
Cognitive skills & -0.193 & 0.015 \\
\hline
\end{tabular}

Regression analysis was then performed between selfassessed outcomes and audiometric outcomes, hearing impairment, cognitive skills, patient age and HA use considered as independent variables. A forward selection with entry criterion was implemented and revealed that cognitive skills $(\mathrm{p}=0.521)$, HA use $(\mathrm{p}=0.690)$ and patient age $(\mathrm{p}=0.180)$ did not directly contribute to the overall regression model, whereas the remaining independent variables - audiometric outcomes and hearing impairment - significantly predicted self-assessed outcomes $\left(\mathrm{R}^{2}=0.131\right.$, adjusted $\mathrm{R}^{2}=0.113, \mathrm{~F}(2,97)=7.308$, $\mathrm{p}<0.01$ ). As summarised in Table $\mathrm{V}$, audiometric outcomes and hearing impairment gave almost the same contribution to the self-assessed outcomes (standardised coefficient 0.211 for the audiometric outcomes and 0.205 for hearing impairment); the significance level was $\mathrm{p}<0.10$. Cognitive skills and subject's age contributed only indirectly to self-assessed outcomes through their correlation with audiometric outcomes (see Table IV).

\section{Discussion}

The sample population was large enough to give a reliable picture of the benefit from HA use in the elderly. Most subjects consistently use HAs during the day for at least 8 hours. Differently from similar studies, see e.g. Solheim et al. ${ }^{23}$, the duration of HA use was not associated with the degree of hearing impairment. It is, however, to note that the average hearing impairment in our sample population was greater than that observed in the study cited above because subjects participating in the study were recruited among those eligible for HA provision by the Italian National Health System, which does not cover HA costs if hearing impairment is of a slight degree. This might have biased our results towards the higher range of hearing impairment for which it is expected to have a higher duration of HA use. Consistently with Solheim et al. ${ }^{23}$, no significant relation was observed between HA use and subject's age.

\section{Audiometric outcomes in aided conditions}

Amplified thresholds in sound-field were on average well within the levels of the average speech spectrum at

Table V. The regression model between self-assessed outcomes and the predictors, i.e., audiometric outcomes and hearing impairment (4FAHL).

\begin{tabular}{lcc} 
Predictors & Standardised coefficients & $\mathbf{p}$ \\
Constant & - & 0.070 \\
Audiometric outcomes & 0.211 & 0.059 \\
Hearing impairment & 0.205 & 0.066 \\
\hline
\end{tabular}


$65 \mathrm{~dB}$ SPL. Aided speech reception in quiet (SRT) was for nearly half of the subjects within $50 \mathrm{~dB}$. For more than half of the subjects (61\%), SRT-noise was within $+10 \mathrm{~dB}$ S/N: this result is in line with similar findings in adults and older adults fitted with HAs ${ }^{24}$.

\section{Self-assessed outcomes}

This study supports the substantial improvement in selfassessed outcomes of aged HA users as reported in previous works ${ }^{10}{ }^{11}$. For IOI-HA, scores were equal or even greater than those observed in the norm population ${ }^{20}$, indicating that the level of satisfaction with HA of the participating subjects was in line or even better than that expected in the average population of HA users ${ }^{724}$. As revealed by HHIE-S, most subjects reported to have a mild or non-significant residual hearing handicap in the aided condition. HHIE-S scores in our population were close to the typical findings from HHIE assessment in HA users with similar age and degree of hearing impairment ${ }^{26}$. One study ${ }^{27}$ reported slightly better outcomes; however, subjects involved in that study were slightly younger than ours and with a lower degree of hearing impairment.

The analysis of APHAB scores revealed that the percentage of problems perceived by the subjects diminished in the aided condition compared to the unaided one. This improvement was perceived in all APHAB scales, except the "aversiveness to loudness" of environmental sounds, where scores measured in the aided condition were slightly higher than in the unaided condition, which would suggest worsening in the aided condition. Although significant from a statistical point of view, the score difference in this subscale was very marginal and of no clinical interest. Our results confirmed previous findings reporting a significant reduction in the perceived communication difficulties in the aided elderly population as measured with APHAB (see e.g. McArdle et al. ${ }^{27}$ ).

\section{Factors affecting aided audiometric \\ and self-assessed outcomes}

As a general remark, our results support a significant association between auditory outcomes, hearing impairment and cognition. The impact of cognition might be interpreted as if subjects performing better in cognitive tasks were able to gain better benefit in HA use ${ }^{28}$. A number of studies reported a direct contribution of cognition and/or hearing impairment on audiometric outcomes in HA users ${ }^{568}$. In other studies, an indirect ${ }^{7}$ or no association ${ }^{4}$ between aided audiometric outcomes and cognition was observed. It is to note, however, that the results on the predictive value of cognitive tests on aided speech recognition have to be interpreted with caution because, as discussed by Foo et al. ${ }^{29}$, the predictive value of a cognitive test depends on the type of the test and the settings of the HA.

Differently from previous findings (see e.g. Lunner ${ }^{5}$, Lunner and Sundewall-Thorén ${ }^{6}$, Meister et al. ${ }^{7}$, Rönnberg et al. ${ }^{8}$, Moore et al. ${ }^{9}$ ), no significant correlation was found between age and cognitive skills neither between age and the degree of hearing impairment. It is, however, to note that the age of the population analysed was narrowly distributed: this could have diminished the chance to observe significant variations in cognitive abilities and hearing impairment degree among the subjects.

The analysis also revealed that hearing impairment and audiometric outcomes were significant contributors to self-assessed outcomes, whereas cognitive skills and age contributed to self-assessed outcomes only indirectly, i.e., through their relationship with audiometric outcomes. These findings correlate well with LopezPoveda ${ }^{4}$ and further support the role of aided audiometric outcomes on self-assessed outcomes (i.e., on the perceived benefit of hearing ability).

\section{Conclusions}

The current study provides a "snapshot" on the performance of HA use in a sample population of elderly experienced HA users by measuring both audiometric and self-assessed outcomes. A significant association was observed between hearing impairment and cognitive skills with aided audiometric outcomes, showing that subjects with a lower degree of hearing impairment gained better audiometric outcomes with HA; cognitive skills significantly correlated with audiometric outcomes, but to a lesser extent than hearing impairment. Furthermore, it was also found that self-assessed outcomes were significantly correlated with aided audiometric outcomes and hearing impairment, as subjects with better audiometric outcomes and lower hearing impairment perceived reported higher self-assessed outcomes in using their HAs. Cognitive skills were found to be only indirectly associated with self-assessed outcomes through their direct correlation with aided audiometric outcomes.

This study did not investigate the role played by HA use on preserving long-term health outcomes such as cognitive function: this objective was out of the scope of the present work and would require a different study design involving longitudinal outcome measurements. A number of studies are currently in progress on this topic and also on new HA devices and innovative way to assess PTA 
(see, e.g. Dawes et al. ${ }^{12} 30$, Berrettini et al. ${ }^{31}$; Aimoni et al. ${ }^{32}$ ). Determining the direction of association between cognition and HA benefit still remains an open question, that is whether better cognition causes better HA benefit, or vice versa whether better HA benefit causes better cognitive abilities in a longitudinal perspective.

\section{Acknowledgements}

The authors wish to thank Alessandra Murri (Hospital "G. Saliceto", Piacenza) for useful comments and revisions provided during preparation of this paper, Valeria Castelli (Hospital "G. Saliceto", Piacenza) for the support in clinical data measurement and Sara Colombi (University of Parma, ENT Clinic) for clinical data management.

This work was supported by the "PNRCNR Aging Program 2012-2018".

\section{Conflict of interest statement}

None declared.

\section{References}

1 World Health Organization. WHO global estimates on prevalence of hearing loss. 2012.

2 Fortunato S, Forli F, Guglielmi V, et al. A review of new insights on the association between hearing loss and cognitive decline in ageing. Acta Otorhinolaryngol Ital 2016;36:155-66. https://doi. org/10.14639/0392-100X-993

3 Souza P, Arehart K, Neher T. Working memory and hearing aid processing: Literature findings, future directions, and clinical applications. Front Psychol 2015;6:1894. https://doi.org/10.3389/fpsyg.2015.01894

4 Lopez-PovedaEA, Johannesen PT, Pérez-GonzálezP, etal. Predictors of hearing-aid outcomes. Trends Hear 2017;21:2331216517730526. https://doi.org/10.1177/2331216517730526

5 Lunner T. Cognitive function in relation to hearing aid use. Int $\mathrm{J}$ Audiol 2003;42(Suppl 1):49-58.

6 Lunner T, Sundewall-Thorén E. Interactions between cognition, compression, and listening conditions: effects on speech-in-noise performance in a two-channel hearing aid. J Am Acad Audiol 2007;18:604-17.

7 Meister H, Rählmann S, Walger M, et al. Hearing aid fitting in older persons with hearing impairment: the influence of cognitive function, age, and hearing loss on hearing aid benefit. Clin Interv Aging 2015;10:435-43. https://doi.org/10.2147/CIA.S77096

8 Rönnberg J, Lunner T, Ng EH, et al. Hearing impairment, cognition and speech understanding: exploratory factor analyses of a comprehensive test battery for a group of hearing aid users, the n200 study. Int J Audiol 2016;55:623-42. https://doi.org/10.1080/14992 027.2016.1219775

9 Moore D, Edmondson-Jones M, Dawes P, et al. Relation between speech-in-noise threshold, hearing loss and cognition from 40-69 years of age. PLoS One 2014;9:e107720. https://doi.org/10.1371/ journal.pone.0107720
10 Ferguson M, Kitterick P, Chong L, et al. Hearing aids for mild to moderate hearing loss in adults. Cochrane Database Syst Rev 2017;9:CD012023. https://doi.org/10.1002/14651858.CD012023. pub2

11 Chisolm T, Johnson C, Danhauer J, et al. A systematic review of health-related quality of life and hearing aids: final report of the American Academy of Audiology Task Force on the health-related quality of life benefits of amplification in adults. J Am Acad Audiol 2007; 18:151-83.

12 Dawes P, Cruickshanks K, Fischer M, et al. Hearing-aid use and long-term health outcomes: hearing handicap, mental health, social engagement, cognitive function, physical health, and mortality. Int J Audiol 2015;54:838-44. https://doi.org/10 $.3109 / 14992027.2015 .1059503$

13 Turrini M, Cutugno F, Maturi P, et al. Bisyllabic words for speech audiometry: a new Italian material. Acta Otorhinolaryngol Ital 1993;13:63-77.

14 Puglisi G, Warzybok A, Hochmuth S, et al. An Italian matrix sentence test for the evaluation of speech intelligibility in noise. Int $\mathrm{J}$ Audiol 2015;54(Suppl 2):44-50. https://doi.org/10.3109/14992027. 2015.1061709

15 Kollmeier B, Warzybok A, Hochmuth S, et al. The multilingual matrix test: Principles, applications, and comparison across languages: a review. Int J Audiol 2015;54(Suppl 2):3-16. https://doi.org/10.3109/1 4992027.2015.1020971

16 Cox R, Alexander G. The International Outcome Inventory for Hearing Aids (IOI-HA): psychometric properties of the English version. Int J Audiol 2002;41:30-5.

17 Ventry I, Weinstein B. Identification of elderly people with hearing problems. ASHA 1983;25:37-42.

18 Cox R, Alexander G. The abbreviated profile of hearing aid benefit. Ear Hear 1995;16:176-86. https://doi.org/10.1097/00003446199504000-00005

19 Nasreddine Z, Phillips N, Bédirian V, et al. The Montreal Cognitive Assessment, MoCA: a brief screening tool for mild cognitive impairment. J Am Geriatr Soc 2005;53:695-9. https://doi. org/10.1111/j.1532-5415.2005.53221.x

20 Cox R, Alexander G, Beyer C. Norms for the international outcome inventory for hearing aids. J Am Acad Audiol 2003;14:403-13.

21 Lichtenstein MJ, Bess FH, Logan SA. Validation of screening tools for identifying hearing-impaired elderly in primary care. JAMA 1988;259:2875-8.

22 Santangelo G, Siciliano M, Pedone R, et al. Normative data for the Montreal Cognitive Assessment in an Italian population sample. Neurol Sci 2015;36:585-91. https://doi.org/10.1007/s10072-0141995-y

23 Solheim J, Gay C, Hickson L. Older adults' experiences and issues with hearing aids in the first six months after hearing aid fitting. Int J Audiol 2017;31-9. https://doi.org/10.1080/14992027.2017.13 80849

24 Bohnert A, Nyffeler M, Keilmann A. Advantages of a non-linear frequency compression algorithm in noise. Eur Arch Otorhinolaryngol 2010;267:1045-53. https://doi.org/10.1007/s00405-009-1170-x

25 Smith S, Noe C, Alexander G. Evaluation of the international outcome inventory for hearing aids in a veteran sample. J Am Acad Audiol 2009;20:374-80.

26 Vuorialho A, Karinen P, Sorri M. Effect of hearing aids on hearing disability and quality of life in the elderly. Int J Audiol 2006;45:4005. https://doi.org/10.1080/14992020600625007

27 McArdle R, Chisolm T, Abrams H, et al. The WHO-DAS II: measuring outcomes of hearing aid intervention for adults. Trends Hear 2005;9:127-43. 
G. Tognola et al.

28 Gatehouse S, Naylor G, Elberling C. Benefits from hearing aids in relation to the interaction between the user and the environment. Int J Audiol 2003;42(Suppl 1):77-85.

29 Foo C, Rudner M, Rönnberg J, et al. Recognition of speech in noise with new hearing instrument compression release settings requires explicit cognitive storage and processing capacity. J Am Acad Audiol 2007;18:618-31.

30 Dawes P, Emsley R, Cruickshanks K, et al. Hearing loss and cognition: the role of hearing aids, social isolation and depression.
PLoS One 2015;10:e0119616. https://doi.org/10.1371/journal. pone.0119616

31 Berrettini S, Bruschini L, DE Vito A, et al. Transtympanic Hearing Aid: exploratory study on a new device. Acta Otorhinolaryngol Ital 2018;38:236-41. https://doi.org/10.14639/0392-100X-1612

32 Aimoni C, Crema L, Savini S, et al. Hearing threshold estimation by auditory steady state responses (ASSR) in children. Acta Otorhinolaryngol Ital 2018;38:361-8. https://doi.org/10.14639/0392$100 \mathrm{X}-1463$

Received: March 29, 2018 - Accepted: June 12, 2018

How to cite this article: Tognola G, Mainardi A, Vincenti V, et al. Benefit of hearing aid use in the elderly: the impact of age, cognition and hearing impairment. Acta Otorhinolaryngol Ital 2019;39:409-418. https://doi.org/10.14639/0392-100X-2165

Address for correspondence: Gabriella Tognola, CNR IEIIT Istituto di Elettronica e di Ingegneria dell'Informazione e delle Telecomunicazioni, c/o Politecnico di Milano (DEIB), piazza L. da Vinci 32, 20133 Milan, Italy. E-mail: gabriella.tognola@ieiit.cnr.it 